\title{
BOUNDARY VALUE PROBLEMS WITH LOCAL GENERALIZED NEVANLINNA FUNCTIONS IN THE BOUNDARY CONDITION
}

\author{
Jussi Behrndt and Peter Jonas
}

Preprint No. 2004/01 


\title{
BOUNDARY VALUE PROBLEMS WITH LOCAL GENERALIZED NEVANLINNA FUNCTIONS IN THE BOUNDARY CONDITION
}

\author{
Jussi Behrndt and Peter Jonas
}

\begin{abstract}
For a class of abstract $\lambda$-dependent boundary value problems where a local variant of generalized Nevanlinna functions appears in the boundary condition, linearizations are constructed and their local spectral properties are investigated.
\end{abstract}

Keywords. boundary value problems, symmetric and selfadjoint operators and relations in Krein spaces, generalized Nevanlinna functions, boundary value spaces, Weyl functions.

2000 Mathematics Subject Classification. Primary: 47 B 50, 34 B 07; Secondary: 46 C 20, 47 A 06, 47 B 40

\section{INTRODUCTION}

In this paper we study a class of abstract $\lambda$-dependent boundary value problems with a local variant of generalized Nevanlinna functions appearing in the boundary condition. For this let $A$ be a closed symmetric operator or relation with defect one in a separable Krein space $\mathcal{H}$ and let $\left\{\mathbb{C}, \Gamma_{0}, \Gamma_{1}\right\}$ be a boundary value space for the adjoint relation $A^{+}$. We assume that the selfadjoint extension $A_{0}:=\operatorname{ker} \Gamma_{0}$ of $A$ admits a spectral decomposition into two relations one of which acts in a Pontryagin space. A selfadjoint relation with this property is called locally of type $\pi_{+}$(see Definition 3.2). Let $\tau$ be a function which can be written as a sum of a generalized Nevanlinna function and a locally holomorphic function; a so-called local generalized Nevanlinna function (see Definition 3.1). In Theorem 4.1 we investigate boundary value problems of the following form: For a given $h \in \mathcal{H}$ find a vector $\hat{f}=\left(\begin{array}{c}f \\ f^{\prime}\end{array}\right) \in A^{+}$such that

$$
f^{\prime}-\lambda f=h \quad \text { and } \quad \tau(\lambda) \Gamma_{0} \hat{f}+\Gamma_{1} \hat{f}=0
$$

holds. For a suitable $\lambda \in \mathbb{C}$ a solution of this boundary value problem can be obtained with the help of the compressed resolvent of a selfadjoint extension $\widetilde{A}$ of $A$ which acts in a larger Krein space $\mathcal{H} \times \mathcal{K}$, i.e. $f=\left.P_{\mathcal{H}}(\widetilde{A}-\lambda)^{-1}\right|_{\mathcal{H}} h, f^{\prime}=\lambda f+h$ fulfil (1.1). The relation $\widetilde{A}$ is called a linearization of (1.1). We construct $\widetilde{A}$ and investigate its local 
spectral properties, which are closely connected with the solvability of (1.1), with the help of the coupling method from $[7, \S 5.2]$ and a perturbation result from [2]. Here we obtain that $\widetilde{A}$ is locally of type $\pi_{+}$.

We briefly describe the contents of this paper. In Section 2 we recall some basic facts on boundary value spaces and Weyl functions associated with symmetric relations in Krein spaces. In Section 3 it is shown that a local generalized Nevanlinna function can be expressed as the Weyl function corresponding to a symmetric relation and a suitable boundary value space $\left\{\mathbb{C}, \Gamma_{0}^{\prime}, \Gamma_{1}^{\prime}\right\}$ where the selfadjoint relation ker $\Gamma_{0}^{\prime}$ is locally of type $\pi_{+}$. Section 4 contains our main result. Based on the approach in [7] we construct a linearization of the boundary value problem (1.1) which again is locally of type $\pi_{+}$. Under an additional assumption this linearization fulfils a minimality condition. In this case the linearization is, roughly speaking, locally uniquely determined up to unitary equivalence (Remark 4.4).

In a subsequent paper the results obtained in Section 4 will be applied to boundary value problems for differential operators with an indefinite weight.

\section{Boundary Value Spaces and Weyl Functions Associated with a Symmetric Relation in a Krein Space}

Let $(\mathcal{K},[\cdot, \cdot])$ be a separable Krein space with a corresponding fundamental symmetry $J$. The linear space of bounded linear operators defined on a Krein space $\mathcal{K}_{1}$ with values in a Krein space $\mathcal{K}_{2}$ is denoted by $\mathcal{L}\left(\mathcal{K}_{1}, \mathcal{K}_{2}\right)$. If $\mathcal{K}:=\mathcal{K}_{1}=\mathcal{K}_{2}$ we simply write $\mathcal{L}(\mathcal{K})$. We study linear relations in $\mathcal{K}$, that is, linear subspaces of $\mathcal{K}^{2}$. The set of all closed linear relations in $\mathcal{K}$ is denoted by $\widetilde{\mathcal{C}}(\mathcal{K})$. Linear operators in $\mathcal{K}$ are viewed as linear relations via their graphs. For the usual definitions of the linear operations with relations, the inverse etc., we refer to [8]. The sum and the direct sum of subspaces in $\mathcal{K}^{2}$ is denoted by $\boldsymbol{+}$ and $\dot{\boldsymbol{+}}$. We define an indefinite inner product on $\mathcal{K}^{2}$ by

$$
\llbracket \hat{f}, \hat{g} \rrbracket=i\left(\left[f, g^{\prime}\right]-\left[f^{\prime}, g\right]\right), \quad \hat{f}=\left(\begin{array}{c}
f \\
f^{\prime}
\end{array}\right), \hat{g}=\left(\begin{array}{c}
g \\
g^{\prime}
\end{array}\right) \in \mathcal{K}^{2} .
$$

Then $\left(\mathcal{K}^{2}, \llbracket \cdot, \cdot \rrbracket\right)$ is a Krein space and $\mathcal{J}=\left(\begin{array}{cc}0 & -i J \\ i J & 0\end{array}\right) \in \mathcal{L}\left(\mathcal{K}^{2}\right)$ is a corresponding fundamental symmetry. Observe that also in the special case when $(\mathcal{K},[\cdot, \cdot])$ is a Hilbert space, $\llbracket \cdot, \cdot \rrbracket$ is an indefinite metric. In the following we shall use at the same time inner products $\llbracket \cdot, \cdot \rrbracket$ arising from different Krein and Hilbert spaces as in (2.1). Then we shall indicate these forms by subscripts, for example, $\llbracket \cdot, \cdot \rrbracket_{\mathcal{K}^{2}}, \llbracket \cdot, \cdot \rrbracket_{\mathcal{G}^{2}}$.

Let $S$ be a linear relation in $\mathcal{K}$. The adjoint relation $S^{+}$is defined as

$$
S^{\llbracket \perp \rrbracket}=\left\{\hat{h} \in \mathcal{K}^{2} \mid \llbracket \hat{h}, \hat{f} \rrbracket=0 \text { for all } \hat{f} \in S\right\} .
$$

$S$ is said to be symmetric (selfadjoint) if $S \subset S^{+}$(resp. $S=S^{+}$). The resolvent set $\rho(S)$ of $S \in \widetilde{\mathcal{C}}(\mathcal{K})$ is the set of all $\lambda \in \mathbb{C}$ such that $(S-\lambda)^{-1} \in \mathcal{L}(\mathcal{K})$, the spectrum $\sigma(S)$ of $S$ is the complement of $\rho(S)$ in $\mathbb{C}$. For the definition of the point spectrum $\sigma_{p}(S)$, continuous spectrum $\sigma_{c}(S)$ and residual spectrum $\sigma_{r}(S)$ we refer to [8]. The extended spectrum $\widetilde{\sigma}(S)$ of $S$ is defined by $\widetilde{\sigma}(S)=\sigma(S)$ if $S \in \mathcal{L}(\mathcal{K})$ and $\widetilde{\sigma}(S)=\sigma(S) \cup\{\infty\}$ otherwise. 
We say that a closed symmetric relation $A$ has defect $m \in \mathbb{N} \cup\{\infty\}$, if both deficiency indices

$$
n_{ \pm}(J A)=\operatorname{dim} \operatorname{ker}\left((J A)^{*}-\bar{\lambda}\right), \quad \lambda \in \mathbb{C}^{ \pm},
$$

of the symmetric relation $J A$ in the Hilbert space $(\mathcal{K},[J \cdot, \cdot])$ are equal to $m$. With the help of the von Neumann formulas for a closed symmetric relation in a Hilbert space (see e.g. $[6, \S 2.3]$ ) one can verify without difficulty that this is equivalent to the fact that there exists a selfadjoint extension of $A$ in $\mathcal{K}$ and that each selfadjoint extension $\hat{A}$ of $A$ in $\mathcal{K}$ satisfies $\operatorname{dim}(\hat{A} / A)=m$.

We shall use the so-called boundary value spaces for the description of the selfadjoint extensions of closed symmetric relations in Krein spaces. The following definition is taken from [4].

Definition 2.1. Let $A$ be a closed symmetric relation in the Krein space $\mathcal{K}$. We say that $\left\{\mathcal{G}, \Gamma_{0}, \Gamma_{1}\right\}$ is a boundary value space for $A^{+}$if $\mathcal{G}$ is a Hilbert space and there exist mappings $\Gamma_{0}, \Gamma_{1}: A^{+} \rightarrow \mathcal{G}$ such that $\Gamma:=\left(\begin{array}{c}\Gamma_{0} \\ \Gamma_{1}\end{array}\right): A^{+} \rightarrow \mathcal{G}^{2}$ is surjective, and the relation $\llbracket \Gamma \hat{f}, \Gamma \hat{g} \rrbracket_{\mathcal{G}^{2}}=\llbracket \hat{f}, \hat{g} \rrbracket_{\mathcal{K}^{2}}$ holds for all $\hat{f}, \hat{g} \in A^{+}$.

In the following we recall some basic facts on boundary value spaces which can be found in e.g. [3] and [4]. For the Hilbert space case we refer to [9], [5] and [6]. Let $A$, $\left\{\mathcal{G}, \Gamma_{0}, \Gamma_{1}\right\}$ and $\Gamma$ be as in Definition 2.1. It follows that the mappings $\Gamma_{0}$ and $\Gamma_{1}$ are continuous. The selfadjoint extensions

$$
A_{0}:=\operatorname{ker} \Gamma_{0} \quad \text { and } \quad A_{1}:=\operatorname{ker} \Gamma_{1}
$$

of $A$ are transversal, that is $A_{0} \cap A_{1}=A$ and $A_{0}+A_{1}=A^{+}$. The mapping $\Gamma$ induces, via

$$
A_{\Theta}:=\Gamma^{-1} \Theta=\left\{\hat{f} \in A^{+} \mid \Gamma \hat{f} \in \Theta\right\}, \quad \Theta \in \widetilde{\mathcal{C}}(\mathcal{G}),
$$

a bijective correspondence $\Theta \mapsto A_{\Theta}$ between the set of all closed linear relations $\widetilde{\mathcal{C}}(\mathcal{G})$ in $\mathcal{G}$ and the set of closed extensions $A_{\Theta} \subset A^{+}$of $A$. In particular (2.2) gives a one-to-one correspondence between the symmetric (selfadjoint) extensions of $A$ and the symmetric (resp. selfadjoint) relations in $\mathcal{G}$. If $\Theta$ is a closed operator in $\mathcal{G}$, then the corresponding extension $A_{\Theta}$ of $A$ is determined by

$$
A_{\Theta}=\operatorname{ker}\left(\Gamma_{1}-\Theta \Gamma_{0}\right) .
$$

Let again $A$ be a closed symmetric relation in $\mathcal{K}$, let $\left\{\mathcal{G}, \Gamma_{0}, \Gamma_{1}\right\}$ be a boundary value space for $A^{+}$and assume that $A_{0}=\operatorname{ker} \Gamma_{0}$ has a nonempty resolvent set. Let

$$
\mathcal{N}_{\lambda, A^{+}}:=\operatorname{ker}\left(A^{+}-\lambda\right)=\operatorname{ran}(A-\bar{\lambda})^{[\perp]}
$$

be the defect subspace of $A$ and let

$$
\hat{\mathcal{N}}_{\lambda, A^{+}}=\left\{\left(\begin{array}{c}
f_{\lambda} \\
\lambda f_{\lambda}
\end{array}\right) \mid f_{\lambda} \in \mathcal{N}_{\lambda, A^{+}}\right\}
$$

When no confusion can arise we will simply write $\mathcal{N}_{\lambda}$ and $\hat{\mathcal{N}}_{\lambda}$ instead of $\mathcal{N}_{\lambda, A^{+}}$and $\hat{\mathcal{N}}_{\lambda, A^{+}}$. We have

$$
A^{+}=A_{0} \dot{\mathbf{+}} \hat{\mathcal{N}_{\lambda}} \quad \text { for all } \quad \lambda \in \rho\left(A_{0}\right)
$$


(see e.g. [4]). By $\pi_{1}$ we denote the orthogonal projection onto the first component of $\mathcal{K}^{2}$. For every $\lambda \in \rho\left(A_{0}\right)$ we define the operators

$$
\gamma(\lambda)=\pi_{1}\left(\Gamma_{0} \mid \hat{\mathcal{N}}_{\lambda}\right)^{-1} \in \mathcal{L}(\mathcal{G}, \mathcal{K}) \text { and } M(\lambda)=\Gamma_{1}\left(\Gamma_{0} \mid \hat{\mathcal{N}}_{\lambda}\right)^{-1} \in \mathcal{L}(\mathcal{G})
$$

The functions $\lambda \mapsto \gamma(\lambda)$ and $\lambda \mapsto M(\lambda)$ are called the $\gamma$-field and Weyl function corresponding to $A$ and $\left\{\mathcal{G}, \Gamma_{0}, \Gamma_{1}\right\}$. $\gamma$ and $M$ are holomorphic on $\rho\left(A_{0}\right)$ and the relations

$$
\gamma(\zeta)=\left(1+(\zeta-\lambda)\left(A_{0}-\zeta\right)^{-1}\right) \gamma(\lambda)
$$

and

$$
M(\lambda)-M(\zeta)^{*}=(\lambda-\bar{\zeta}) \gamma(\zeta)^{+} \gamma(\lambda)
$$

hold for $\lambda, \zeta \in \rho\left(A_{0}\right)$ (see e.g. [4]). A little calculation yields

$$
M(\lambda)=\operatorname{Re} M\left(\lambda_{0}\right)+\gamma\left(\lambda_{0}\right)^{+}\left(\lambda-\operatorname{Re} \lambda_{0}+\left(\lambda-\lambda_{0}\right)\left(\lambda-\bar{\lambda}_{0}\right)\left(A_{0}-\lambda\right)^{-1}\right) \gamma\left(\lambda_{0}\right)
$$

for all $\lambda \in \rho\left(A_{0}\right)$ and a fixed $\lambda_{0} \in \rho\left(A_{0}\right)$.

The following well-known theorem shows how the spectra of closed extensions of $A$ can be described with the help of the Weyl function. For a proof see e.g. [4].

Theorem 2.2. Let $A$ be a closed symmetric relation in a Krein space $\mathcal{K}$ and let $\left\{\mathcal{G}, \Gamma_{0}, \Gamma_{1}\right\}$ be a boundary value space for $A^{+}$where $A_{0}=\operatorname{ker} \Gamma_{0}$ has a nonempty resolvent set. Denote by $\gamma$ and $M$ the corresponding $\gamma$-field and Weyl function, let $\Theta \in \widetilde{\mathcal{C}}(\mathcal{G})$ and let $A_{\Theta}$ be the corresponding extension. For $\lambda \in \rho\left(A_{0}\right)$ the following assertions are true.

(i) $\lambda \in \sigma_{i}\left(A_{\Theta}\right)$ if and only if $0 \in \sigma_{i}(\Theta-M(\lambda)), i=p, c, r$.

(ii) $\lambda \in \rho\left(A_{\Theta}\right)$ if and only if $0 \in \rho(\Theta-M(\lambda))$.

(iii) For all $\lambda \in \rho\left(A_{\Theta}\right) \cap \rho\left(A_{0}\right)$

$$
\left(A_{\Theta}-\lambda\right)^{-1}=\left(A_{0}-\lambda\right)^{-1}+\gamma(\lambda)(\Theta-M(\lambda))^{-1} \gamma(\bar{\lambda})^{+} .
$$

\section{Local Generalized Nevanlinna Functions as Weyl Functions of Symmetric Relations}

Recall that a piecewise meromorphic function $\tau$ in $\mathbb{C} \backslash \mathbb{R}$ which is symmetric with respect to the real axis (that is $\tau(\bar{\lambda})=\overline{\tau(\lambda)}$ for all points $\lambda$ where $\tau$ is holomorphic) is a generalized Nevanlinna function if the kernel

$$
N_{\tau}(\lambda, \mu):=\frac{\tau(\lambda)-\tau(\bar{\mu})}{\lambda-\bar{\mu}}
$$

has a finite number of negative squares. Here we consider a local variant of generalized Nevanlinna functions ([1, Definition 1.5]). We recall the definition of the class of local generalized Nevanlinna functions, which is a subclass of the class of the so-called locally definitizable functions (see [13]). 
Let $\Omega$ be some domain in $\overline{\mathbb{C}}$ symmetric with respect to the real axis such that $\Omega \cap \overline{\mathbb{R}} \neq \emptyset$ and the intersections of $\Omega$ with the upper and lower open half-planes are simply connected.

Definition 3.1. Let $\tau$ be a piecewise meromorphic function in $\Omega \backslash \overline{\mathbb{R}}$ which is symmetric with respect to the real axis. We say that $\tau$ is a generalized Nevanlinna function over $\Omega$, if for every domain $\Omega^{\prime}$ with the same properties as $\Omega, \overline{\Omega^{\prime}} \subset \Omega, \tau$ can be written in the form

$$
\tau=\tau_{0}+\tau_{(0)},
$$

where $\tau_{0}$ is a generalized Nevanlinna function and $\tau_{(0)}$ is a holomorphic function in $\Omega^{\prime}$. The class of generalized Nevanlinna functions over $\Omega$ is denoted by $N(\Omega)$.

The class $N(\overline{\mathbb{C}})$ coincides with the class of generalized Nevanlinna functions (see [13]). Note, that for $\tau \in N(\Omega)$ the nonreal poles of $\tau$ in $\Omega$ do not accumulate to $\Omega \cap \overline{\mathbb{R}}$. The set of the points of holomorphy of $\tau$ in $\Omega \backslash \overline{\mathbb{R}}$ and all points $\lambda \in \Omega \cap \overline{\mathbb{R}}$ such $\tau$ can be analytically continued to $\lambda$ and the continuations from $\Omega \cap \mathbb{C}^{+}$and $\Omega \cap \mathbb{C}^{-}$coincide, is denoted by $\mathfrak{h}(\tau)$.

In Section 4 below we will make use of the fact that every local generalized Nevanlinna function coincides with the Weyl function corresponding to some boundary value space $\left\{\mathbb{C}, \Gamma_{0}^{\prime}, \Gamma_{1}^{\prime}\right\}$ for a closed symmetric relation where the selfadjoint relation $\operatorname{ker} \Gamma_{0}^{\prime}$ has special spectral properties. For this representation we need the following subclass of locally definitizable selfadjoint relations in a Krein space (see [12]).

Definition 3.2. Let $\Omega$ be a domain as in the beginning of this section and let $A_{0}$ be a selfadjoint relation in the Krein space $(\mathcal{K},[\cdot, \cdot]) . A_{0}$ is said to be of type $\pi_{+}$over $\Omega$ if for every domain $\Omega^{\prime}$ with the same properties as $\Omega, \overline{\Omega^{\prime}} \subset \Omega$, there exists a selfadjoint projection $E$ in $\mathcal{K}$ such that $A$ can be decomposed in

$$
A_{0}=\left(A_{0} \cap(E \mathcal{K})^{2}\right) \dot{+}\left(A_{0} \cap((1-E) \mathcal{K})^{2}\right)
$$

and the following holds.

(i) $E \mathcal{K}$ is a Pontryagin space with finite rank of negativity, $\rho\left(A_{0} \cap(E \mathcal{K})^{2}\right) \neq \emptyset$.

(ii) $\widetilde{\sigma}\left(A_{0} \cap((1-E) \mathcal{K})^{2}\right) \cap \Omega^{\prime}=\emptyset$.

Let $A_{0}$ be a selfadjoint relation of type $\pi_{+}$over $\Omega$ in the Krein space $\mathcal{K}$. Then the set $\tilde{\sigma}\left(A_{0}\right) \cap(\Omega \backslash \overline{\mathbb{R}})$ is discrete and the nonreal spectrum of $A_{0}$ in $\Omega$ does not accumulate to $\Omega \cap \overline{\mathbb{R}}$. Let $\Omega^{\prime}$ be a domain with the same properties as $\Omega, \overline{\Omega^{\prime}} \subset \Omega$, and let $E$ be a selfadjoint projection with the properties as in Definition 3.2. If $E^{\prime}$ is the spectral function of the selfadjoint relation $A_{0} \cap(E \mathcal{K})^{2}$ in the Pontryagin space $E \mathcal{K}$, then the mapping

$$
\delta \mapsto E^{\prime}(\delta) E=: E_{A_{0}}(\delta)
$$

defined for all finite unions $\delta$ of connected subsets of $\Omega^{\prime} \cap \overline{\mathbb{R}}$ the endpoints of which belong to $\Omega^{\prime} \cap \overline{\mathbb{R}}$ and are not critical points of $A_{0} \cap(E \mathcal{K})^{2}$, is the spectral function of $A_{0}$ on $\Omega^{\prime} \cap \overline{\mathbb{R}}$ (see [12, Section 3.4, Remark 4.9]). $E_{A_{0}}(\cdot)$ does not depend on the choice of $E$. 
Let $A \subset A_{0}$ be a closed symmetric relation with defect one and let $\left\{\mathbb{C}, \Gamma_{0}, \Gamma_{1}\right\}$ be a boundary value space for $A^{+}$with $\operatorname{ker} \Gamma_{0}=A_{0}$. We denote the corresponding $\gamma$-field and Weyl function by $\gamma$ and $M$, respectively. Here $\gamma(\lambda) \in \mathcal{L}(\mathbb{C}, \mathcal{K})$ for $\lambda \in \rho\left(A_{0}\right)$, and $M$ is a scalar function. From (2.7) and the assumption on $A_{0}$ we conclude that the Weyl function $M$ can be written as the sum of the generalized Nevanlinna function

$$
M_{0}(\lambda):=\operatorname{Re} M\left(\lambda_{0}\right)+\gamma\left(\lambda_{0}\right)^{+}\left(\lambda-\operatorname{Re} \lambda_{0}+\left(\lambda-\lambda_{0}\right)\left(\lambda-\bar{\lambda}_{0}\right)\left(A_{0}-\lambda\right)^{-1}\right) E \gamma\left(\lambda_{0}\right)
$$

and the function

$$
M_{(0)}(\lambda):=\gamma\left(\lambda_{0}\right)^{+}\left(\lambda-\operatorname{Re} \lambda_{0}+\left(\lambda-\lambda_{0}\right)\left(\lambda-\bar{\lambda}_{0}\right)\left(A_{0}-\lambda\right)^{-1}\right)(1-E) \gamma\left(\lambda_{0}\right)
$$

which is holomorphic in $\Omega^{\prime}$. Therefore, $M \in N(\Omega)$.

Assume now that a function $\tau \in N(\Omega)$ is given. In [13] it was shown that for every domain $\Omega^{\prime}$ with the same properties as $\Omega, \overline{\Omega^{\prime}} \subset \Omega$, there exists a Krein space $(\mathcal{K},[\cdot, \cdot])$, a selfadjoint relation $T_{0}$ in $\mathcal{K}$ of type $\pi_{+}$over $\Omega^{\prime}$ and an element $e \in \mathcal{K}$ such that for a fixed $\lambda_{0} \in \Omega^{\prime} \cap \mathfrak{h}(\tau)$ and every $\lambda \in \Omega^{\prime} \cap \mathfrak{h}(\tau)$ the relation

$$
\left.\tau(\lambda)=\operatorname{Re} \tau\left(\lambda_{0}\right)+\left(\lambda-\operatorname{Re} \lambda_{0}\right)[e, e]+\left(\lambda-\lambda_{0}\right)\left(\lambda-\bar{\lambda}_{0}\right)\left[\left(T_{0}-\lambda\right)^{-1}\right) e, e\right]
$$

holds. Moreover, $\rho\left(T_{0}\right) \cap \Omega^{\prime}=\mathfrak{h}(\tau) \cap \Omega^{\prime}$.

The representation (3.2) is called minimal if

$$
\operatorname{clsp}\left\{\left(1+\left(\lambda-\lambda_{0}\right)\left(T_{0}-\lambda\right)^{-1}\right) e \mid \lambda \in \rho\left(T_{0}\right) \cap \Omega^{\prime}\right\}=\mathcal{K}
$$

holds for some $\lambda_{0} \in \rho\left(T_{0}\right) \cap \Omega^{\prime}$. Such a minimal representation of $\tau$ exists e.g. if, in addition, $\tau$ is the restriction of a generalized Nevanlinna function or a so-called definitizable function (see [10], [11]) to $\Omega^{\prime}$ or if, in addition, the boundary of $\Omega^{\prime}$ is contained in $\mathfrak{h}(\tau)$.

Making use of the representation (3.2) we construct in the following theorem a boundary value space such that $\tau \in N(\Omega)$ is its Weyl function. The idea of the proof is the same as in the proof of [5, Theorem 1].

Theorem 3.3. Let $\Omega$ be as in the beginning of this section and let $\tau \in N(\Omega)$ be nonconstant. Let $\Omega^{\prime}$ be a domain with the same properties as $\Omega, \overline{\Omega^{\prime}} \subset \Omega$, and let $\tau$ be represented with a selfadjoint relation $T_{0}$ of type $\pi_{+}$over $\Omega^{\prime}$ in a Krein space $\mathcal{K}$ as in (3.2). Then there exists a closed symmetric relation $T \subset T_{0}$ with defect one and a boundary value space $\left\{\mathbb{C}, \Gamma_{0}^{\prime}, \Gamma_{1}^{\prime}\right\}$ for $T^{+}$such that $\tau$ coincides with the corresponding Weyl function on $\Omega^{\prime}$.

In the case $\Omega=\overline{\mathbb{C}}$ Theorem 3.3 reads as follows.

Corollary 3.4. Let $\tau$ be a nonconstant generalized Nevanlinna function. Then there exists a closed symmetric relation $T$ in a Pontryagin space $\mathcal{K}$ with finite rank of negativity and a boundary value space $\left\{\mathbb{C}, \Gamma_{0}^{\prime}, \Gamma_{1}^{\prime}\right\}$ for $T^{+}$such that $\tau$ is the corresponding Weyl function.

Proof of Theorem 3.3. The assumption that $\tau$ is not constant implies that the vector $e \in \mathcal{K}$ in the representation (3.2) is not zero. For every $\lambda \in \Omega^{\prime} \cap \mathfrak{h}(\tau)$ and a fixed $\lambda_{0} \in \Omega^{\prime} \cap \mathfrak{h}(\tau)$ we define

$$
\gamma^{\prime}(\lambda):=\left(1+\left(\lambda-\lambda_{0}\right)\left(T_{0}-\lambda\right)^{-1}\right) e,
$$


which implies $\gamma^{\prime}\left(\lambda_{0}\right)=e, \gamma^{\prime}(\zeta)=\left(1+(\zeta-\lambda)\left(T_{0}-\zeta\right)^{-1}\right) \gamma^{\prime}(\lambda)$ and $\gamma^{\prime}(\lambda) \neq 0$ for all $\lambda, \zeta \in \Omega^{\prime} \cap \mathfrak{h}(\tau)$. For some $\mu \in \Omega^{\prime} \cap \mathfrak{h}(\tau)$ we define the closed symmetric relation

$$
T:=\left\{\left(\begin{array}{l}
f \\
g
\end{array}\right) \in T_{0} \mid\left[g-\bar{\mu} f, \gamma^{\prime}(\mu)\right]=0\right\}
$$

in $\mathcal{K}$. As

$$
\left[g-\bar{\mu}^{\prime} f, \gamma^{\prime}\left(\mu^{\prime}\right)\right]=\left[g-\bar{\mu}^{\prime} f,\left(1+\left(\mu^{\prime}-\mu\right)\left(T_{0}-\mu^{\prime}\right)^{-1}\right) \gamma^{\prime}(\mu)\right]=\left[g-\bar{\mu} f, \gamma^{\prime}(\mu)\right]
$$

for all $\left(\begin{array}{c}f \\ g\end{array}\right) \in T_{0}$ and $\mu^{\prime} \in \Omega^{\prime} \cap \mathfrak{h}(\tau)$, the relation $T$ does not depend on the choice of $\mu$. By (3.5) we have $\mathcal{N}_{\mu}=\operatorname{ran}(T-\bar{\mu})^{[\perp]}=\operatorname{sp} \gamma^{\prime}(\mu)$.

Now we regard $\gamma^{\prime}(\lambda), \lambda \in \Omega^{\prime} \cap \mathfrak{h}(\tau)$, as the linear mapping $\mathbb{C} \ni c \mapsto c \gamma^{\prime}(\lambda) \in \mathcal{K}$ and denote the linear functional $c \gamma^{\prime}(\lambda) \mapsto c$ defined on $\mathcal{N}_{\lambda}=\operatorname{sp} \gamma^{\prime}(\lambda)$ by $\gamma^{\prime}(\lambda)^{(-1)}$.

We write the elements $\hat{f} \in T^{+}$, for every $\lambda \in \Omega^{\prime} \cap \mathfrak{h}(\tau)$, in the form

$$
\hat{f}=\left(\begin{array}{c}
f_{0} \\
f_{0}^{\prime}
\end{array}\right)+\left(\begin{array}{c}
f_{\lambda} \\
\lambda f_{\lambda}
\end{array}\right)
$$

where $\left(\begin{array}{l}f_{0} \\ f_{0}^{\prime}\end{array}\right) \in T_{0}$ and $f_{\lambda} \in \mathcal{N}_{\lambda}$ (see $\left.(2.5)\right)$. Let $\Gamma_{0}^{\prime}, \Gamma_{1}^{\prime}: T^{+} \rightarrow \mathbb{C}$ be the linear functionals defined by

$$
\begin{aligned}
& \Gamma_{0}^{\prime} \hat{f}:=\gamma^{\prime}(\lambda)^{(-1)} f_{\lambda}, \\
& \Gamma_{1}^{\prime} \hat{f}:=\gamma^{\prime}(\lambda)^{+}\left(f_{0}^{\prime}-\bar{\lambda} f_{0}\right)+\tau(\lambda) \gamma^{\prime}(\lambda)^{(-1)} f_{\lambda} .
\end{aligned}
$$

The mapping $\Gamma^{\prime}:=\left(\begin{array}{c}\Gamma_{0}^{\prime} \\ \Gamma_{1}^{\prime}\end{array}\right): T^{+} \rightarrow \mathbb{C}^{2}$ is surjective. Indeed, let $\left(\begin{array}{l}h_{1} \\ h_{2}\end{array}\right) \in \mathbb{C}^{2}$ and set $f_{\lambda}:=\gamma^{\prime}(\lambda) h_{1} \in \mathcal{N}_{\lambda}$. Since, by the relation $\{0\}=\operatorname{ker} \gamma^{\prime}(\lambda)=\left(\operatorname{ran} \gamma^{\prime}(\lambda)^{+}\right)^{\perp}, \gamma^{\prime}(\lambda)^{+}$is surjective, there exists $\left(\begin{array}{c}f_{0} \\ f_{0}^{\prime}\end{array}\right) \in T_{0}$ such that $\gamma^{\prime}(\lambda)^{+}\left(f_{0}^{\prime}-\bar{\lambda} f_{0}\right)=h_{2}-\tau(\lambda) h_{1}$. Then

$$
\Gamma^{\prime}\left(\left(\begin{array}{c}
f_{0} \\
f_{0}^{\prime}
\end{array}\right)+\left(\begin{array}{c}
f_{\lambda} \\
\lambda f_{\lambda}
\end{array}\right)\right)=\left(\begin{array}{c}
h_{1} \\
h_{2}
\end{array}\right) .
$$

Making use of the relation $\tau(\lambda)-\tau(\bar{\zeta})=(\lambda-\bar{\zeta}) \gamma^{\prime}(\zeta)^{+} \gamma^{\prime}(\lambda)$, which can be verified by a straightforward calculation, we obtain

$$
\begin{aligned}
\llbracket \hat{f}, \hat{g} \rrbracket_{\mathcal{K}^{2}}= & \llbracket\left(\begin{array}{c}
f_{0} \\
f_{0}^{\prime}
\end{array}\right)+\left(\begin{array}{c}
f_{\lambda} \\
\lambda f_{\lambda}
\end{array}\right),\left(\begin{array}{c}
g_{0} \\
g_{0}^{\prime}
\end{array}\right)+\left(\begin{array}{c}
g_{\lambda} \\
\lambda g_{\lambda}
\end{array}\right) \rrbracket_{\mathcal{K}^{2}} \\
= & i\left(\left[f_{\lambda}, g_{0}^{\prime}-\bar{\lambda} g_{0}\right]-\left[f_{0}^{\prime}-\bar{\lambda} f_{0}, g_{\lambda}\right]-\left[(\lambda-\bar{\lambda}) f_{\lambda}, g_{\lambda}\right]\right) \\
= & i\left(\left(\gamma^{\prime}(\lambda)^{(-1)} f_{\lambda}, \gamma^{\prime}(\lambda)^{+}\left(g_{0}^{\prime}-\bar{\lambda} g_{0}\right)\right)-\left(\gamma^{\prime}(\lambda)^{+}\left(f_{0}^{\prime}-\bar{\lambda} f_{0}\right), \gamma^{\prime}(\lambda)^{(-1)} g_{\lambda}\right)\right. \\
& \left.\quad-\left((\tau(\lambda)-\tau(\bar{\lambda})) \gamma^{\prime}(\lambda)^{(-1)} f_{\lambda}, \gamma^{\prime}(\lambda)^{(-1)} g_{\lambda}\right)\right) \\
= & i\left(\left(\gamma^{\prime}(\lambda)^{(-1)} f_{\lambda}, \gamma^{\prime}(\lambda)^{+}\left(g_{0}^{\prime}-\bar{\lambda} g_{0}\right)+\tau(\lambda) \gamma^{\prime}(\lambda)^{(-1)} g_{\lambda}\right)\right. \\
& \left.\quad-\left(\gamma^{\prime}(\lambda)^{+}\left(f_{0}^{\prime}-\bar{\lambda} f_{0}\right)+\tau(\lambda) \gamma^{\prime}(\lambda)^{(-1)} f_{\lambda}, \gamma^{\prime}(\lambda)^{(-1)} g_{\lambda}\right)\right) \\
= & \llbracket \Gamma^{\prime} \hat{f}, \Gamma^{\prime} \hat{g} \rrbracket_{\mathbb{C}^{2}} .
\end{aligned}
$$


Hence $\left\{\mathbb{C}, \Gamma_{0}^{\prime}, \Gamma_{1}^{\prime}\right\}$ is a boundary value space for $T^{+}$. Moreover, we have ker $\Gamma_{0}^{\prime}=T_{0}$ and the corresponding $\gamma$-field coincides with $\gamma^{\prime}$. For $h=\left(\begin{array}{c}h_{\lambda} \\ \lambda h_{\lambda}\end{array}\right) \in \hat{\mathcal{N}}_{\lambda}$ we obtain

$$
\tau(\lambda) \Gamma_{0} h=\tau(\lambda) \gamma^{\prime}(\lambda)^{(-1)} h_{\lambda}=\Gamma_{1} h .
$$

Therefore $\tau$ coincides with the Weyl function of $T$ on $\Omega^{\prime}$ corresponding to the boundary value space $\left\{\mathbb{C}, \Gamma_{0}^{\prime}, \Gamma_{1}^{\prime}\right\}$ defined in (3.6).

\section{Boundary Value Problems with Spectral Parameter in the Boundary CONDITION}

In this section we consider a class of abstract boundary value problems of the form (1.1) where the spectral parameter appears nonlinearly in the boundary condition. Theorem 4.1 and Corollary 4.2 extend results obtained with the help of the coupling method in [7] for a symmetric operator $A$ in a Hilbert space and a Nevanlinna function $\tau$ in the boundary condition. In contrast to [7] we consider only the case where $\tau$ is a scalar function.

Let $\left(\mathcal{H},[\cdot, \cdot]_{\mathcal{H}}\right)$ and $\left(\mathcal{K},[\cdot, \cdot]_{\mathcal{K}}\right)$ be Krein spaces. The elements of $\mathcal{H} \times \mathcal{K}$ will be written in the form $\{h, k\}, h \in \mathcal{H}, k \in \mathcal{K} . \mathcal{H} \times \mathcal{K}$ equipped with the inner product $[\cdot, \cdot]$ defined by

$$
\left[\left\{h_{1}, k_{1}\right\},\left\{h_{2}, k_{2}\right\}\right]:=\left[h_{1}, h_{2}\right]_{\mathcal{H}}+\left[k_{1}, k_{2}\right]_{\mathcal{K}}, \quad h_{1}, h_{2} \in \mathcal{H}, \quad k_{1}, k_{2} \in \mathcal{K},
$$

is a Krein space. If $A$ is a relation in $\mathcal{H}$ and $T$ is a relation in $\mathcal{K}$ we shall write $A \times T$ for the direct product of $A$ and $T$ which is a relation in $\mathcal{H} \times \mathcal{K}$,

$$
A \times T=\left\{\left(\begin{array}{c}
\{a, t\} \\
\left\{a^{\prime}, t^{\prime}\right\}
\end{array}\right) \mid\left(\begin{array}{c}
a \\
a^{\prime}
\end{array}\right) \in A,\left(\begin{array}{c}
t \\
t^{\prime}
\end{array}\right) \in T\right\} .
$$

For the pair $\left(\begin{array}{c}\{a, t\} \\ \left\{a^{\prime}, t^{\prime}\right\}\end{array}\right)$ on the right hand side of (4.1) we shall also write $\{\hat{a}, \hat{t}\}$, where $\hat{a}=\left(\begin{array}{c}a \\ a^{\prime}\end{array}\right), \hat{t}=\left(\begin{array}{c}t \\ t^{\prime}\end{array}\right)$.

Let, as in Section 3, $\Omega$ be some domain in $\overline{\mathbb{C}}$ symmetric with respect to the real axis such that $\Omega \cap \overline{\mathbb{R}} \neq \emptyset$ and the intersections of $\Omega$ with the upper and lower open half-planes are simply connected and let $\Omega^{\prime}$ be a domain with the same properties as $\Omega$ such that $\overline{\Omega^{\prime}} \subset \Omega$.

Theorem 4.1. Let $A$ be a closed symmetric relation with defect one in the Krein space $\mathcal{H}$ and assume that there exists a selfadjoint extension $A_{0}$ of $A$ which is of type $\pi_{+}$over $\Omega$. Let $\left\{\mathbb{C}, \Gamma_{0}, \Gamma_{1}\right\}$ be a boundary value space for $A^{+}, A_{0}=\operatorname{ker} \Gamma_{0}$, and denote by $\gamma$ and $M$ the corresponding $\gamma$-field and the Weyl function, respectively.

Let $\tau \in N(\Omega)$ be nonconstant and assume that $M+\tau$ is not identically equal to zero in $\Omega \backslash \overline{\mathbb{R}}$. Let $T$ be a closed symmetric relation with defect one in a Krein space $\mathcal{K}$ and let $\left\{\mathbb{C}, \Gamma_{0}^{\prime}, \Gamma_{1}^{\prime}\right\}$ be a boundary value space for $T^{+}$such that $\tau$ coincides with the corresponding Weyl function on $\Omega^{\prime}$ and $T_{0}=\operatorname{ker} \Gamma_{0}^{\prime}$ is of type $\pi_{+}$over $\Omega^{\prime}$ (see Theorem 3.3).

Then the relation

$$
\widetilde{A}=\left\{\left\{\hat{f}_{1}, \hat{f}_{2}\right\} \in A^{+} \times T^{+} \mid \Gamma_{1} \hat{f}_{1}-\Gamma_{1}^{\prime} \hat{f}_{2}=\Gamma_{0} \hat{f}_{1}+\Gamma_{0}^{\prime} \hat{f}_{2}=0\right\}
$$


is a selfadjoint extension of $A$ in $\mathcal{H} \times \mathcal{K}$ which is of type $\pi_{+}$over $\Omega^{\prime}$. For every $h \in \mathcal{H}$ and every $\lambda \in \Omega^{\prime} \backslash(\overline{\mathbb{R}} \cup \Sigma)$, where $\Sigma$ is some finite set, a solution of the $\lambda$-dependent boundary value problem

$$
f_{1}^{\prime}-\lambda f_{1}=h, \quad \tau(\lambda) \Gamma_{0} \hat{f}_{1}+\Gamma_{1} \hat{f}_{1}=0, \quad \hat{f}_{1}=\left(\begin{array}{c}
f_{1} \\
f_{1}^{\prime}
\end{array}\right) \in A^{+},
$$

is given by

$$
\begin{aligned}
& f_{1}=P_{\mathcal{H}}(\widetilde{A}-\lambda)^{-1}\{h, 0\}=\left(A_{0}-\lambda\right)^{-1} h-\gamma(\lambda)(M(\lambda)+\tau(\lambda))^{-1} \gamma(\bar{\lambda})^{+} h, \\
& f_{1}^{\prime}=\lambda f_{1}+h .
\end{aligned}
$$

If, in addition, the representation (3.2) of $\tau$ on $\Omega^{\prime}$ is minimal, then $\widetilde{A}$ satisfies the minimality condition

$$
\operatorname{clsp}\left\{\left(1+\left(\lambda-\lambda_{0}\right)(\widetilde{A}-\lambda)^{-1}\right)\{h, 0\} \mid h \in \mathcal{H}, \lambda \in \rho(\widetilde{A}) \cap \Omega^{\prime}\right\}=\mathcal{H} \times \mathcal{K}
$$

for some $\lambda_{0} \in \rho(\widetilde{A}) \cap \Omega^{\prime}$.

In the next corollary we consider the special case that $\mathcal{H}$ is a Pontryagin space and $\tau$ is a generalized Nevanlinna function.

Corollary 4.2. Let $A$ be a closed symmetric relation with defect one in the Pontryagin space $\mathcal{H}$ with finite rank of negativity and assume that there exists a selfadjoint extension $A_{0}$ which has a nonempty resolvent set. Let $\left\{\mathbb{C}, \Gamma_{0}, \Gamma_{1}\right\}$ be a boundary value space for $A^{+}, A_{0}=\operatorname{ker} \Gamma_{0}$, and denote by $\gamma$ and $M$ the corresponding $\gamma$-field and the Weyl function, respectively.

Let $\tau \in N(\overline{\mathbb{C}})$ be nonconstant and assume that $M+\tau$ is not identically equal to zero. Let $T_{0}$ be a minimal representing relation for $\tau$ in a Pontryagin space $\mathcal{K}$, let $T \subset T_{0}$ be a closed symmetric relation with defect one and let $\left\{\mathbb{C}, \Gamma_{0}^{\prime}, \Gamma_{1}^{\prime}\right\}$ be a boundary value space for $T^{+}$such that $\tau$ is the corresponding Weyl function and $T_{0}=\operatorname{ker} \Gamma_{0}^{\prime}$ (see Corollary 3.4).

Then the relation (4.2) is a selfadjoint extension of $A$ in the Pontryagin space $\mathcal{H} \times \mathcal{K}$, and $\widetilde{A}$ is minimal, that is (4.5) holds with $\rho(\widetilde{A}) \cap \Omega^{\prime}$ replaced by $\rho(\widetilde{A})$. For every $h \in \mathcal{H}$ and every $\lambda \in \mathbb{C} \backslash(\mathbb{R} \cup \Sigma)$, where $\Sigma$ is a finite set, a solution of the $\lambda$-dependent boundary value problem (4.3) is given by (4.4).

Proof of Theorem 4.1. As was shown below Definition 3.2 the Weyl function $M$ corresponding to the boundary value space $\left\{\mathbb{C}, \Gamma_{0}, \Gamma_{1}\right\}$ is a generalized Nevanlinna function in $\Omega$. Since $\tau$ and $M+\tau$ belong to the class $N(\Omega)$ [1, Theorem 2.2] implies that $-\tau^{-1}$ and $-(M+\tau)^{-1}$ belong also to $N(\Omega)$. Therefore their nonreal poles in $\Omega$ do not accumulate to $\Omega \cap \overline{\mathbb{R}}$ and we conclude that the set

$$
\Sigma:=\left\{\mu \in \Omega^{\prime} \backslash \overline{\mathbb{R}} \mid \mu \notin \mathfrak{h}(M) \cup \mathfrak{h}(\tau) \cup \mathfrak{h}\left(\tau^{-1}\right) \cup \mathfrak{h}\left((M+\tau)^{-1}\right)\right\}
$$

is finite. We define the set $\mathfrak{h}_{0}:=\mathfrak{h}(M) \cap \mathfrak{h}(\tau) \cap \mathfrak{h}\left(\tau^{-1}\right) \cap \mathfrak{h}\left((M+\tau)^{-1}\right)$. Note, that $\left(\Omega^{\prime} \backslash \overline{\mathbb{R}}\right) \cap \mathfrak{h}_{0}$ coincides with $\Omega^{\prime} \backslash(\overline{\mathbb{R}} \cup \Sigma)$.

Let $\mathcal{K}, T \subset T_{0} \subset T^{+}$and $\left\{\mathbb{C}, \Gamma_{0}^{\prime}, \Gamma_{1}^{\prime}\right\}$ be as in the assumptions of the theorem, $T_{1}:=\operatorname{ker} \Gamma_{1}^{\prime}$ and let $\gamma^{\prime}$ be the $\gamma$-field corresponding to $\left\{\mathbb{C}, \Gamma_{0}^{\prime}, \Gamma_{1}^{\prime}\right\}$. We define the 
mappings $\widetilde{\Gamma}_{0}, \widetilde{\Gamma}_{1}: A^{+} \times T^{+} \rightarrow \mathbb{C}^{2}$ by

$$
\widetilde{\Gamma}_{0}=\left(\begin{array}{cc}
\Gamma_{0} & 0 \\
0 & \Gamma_{1}^{\prime}
\end{array}\right) \quad \text { and } \quad \widetilde{\Gamma}_{1}=\left(\begin{array}{cc}
\Gamma_{1} & 0 \\
0 & -\Gamma_{0}^{\prime}
\end{array}\right)
$$

It is easy to see that $\left\{\mathbb{C}^{2}, \widetilde{\Gamma}_{0}, \widetilde{\Gamma}_{1}\right\}$ is a boundary value space for $A^{+} \times T^{+}$. On account of $\hat{\mathcal{N}}_{\lambda, A^{+} \times T^{+}}=\hat{\mathcal{N}}_{\lambda, A^{+}} \times \hat{\mathcal{N}}_{\lambda, T^{+}}$(see (2.4)), it follows that the $\gamma$-field $\widetilde{\gamma}$ corresponding to $\left\{\mathbb{C}^{2}, \widetilde{\Gamma}_{0}, \widetilde{\Gamma}_{1}\right\}$ is given by

$$
\widetilde{\gamma}(\lambda)=\left(\begin{array}{cc}
\gamma(\lambda) & 0 \\
0 & \gamma^{\prime}(\lambda) \tau(\lambda)^{-1}
\end{array}\right), \quad \lambda \in \mathfrak{h}(M) \cap \mathfrak{h}(\tau) \cap \mathfrak{h}\left(\tau^{-1}\right),
$$

and the corresponding Weyl function $\widetilde{M}$ is

$$
\widetilde{M}(\lambda)=\left(\begin{array}{cc}
M(\lambda) & 0 \\
0 & -\tau(\lambda)^{-1}
\end{array}\right), \quad \lambda \in \mathfrak{h}(M) \cap \mathfrak{h}(\tau) \cap \mathfrak{h}\left(\tau^{-1}\right),
$$

(see $(2.6))$.

The selfadjoint relation $\widetilde{A}$ in $\mathcal{H} \times \mathcal{K}$ corresponding to $\Theta:=\left(\begin{array}{ll}0 & 1 \\ 1 & 0\end{array}\right) \in \mathcal{L}\left(\mathbb{C}^{2}\right)$ via $(2.2)$, (2.3) is given by

$$
\widetilde{A}=\operatorname{ker}\left(\widetilde{\Gamma}_{1}-\Theta \widetilde{\Gamma}_{0}\right)=\left\{\left\{\hat{f}_{1}, \hat{f}_{2}\right\} \in A^{+} \times T^{+} \mid \Gamma_{1} \hat{f}_{1}-\Gamma_{1}^{\prime} \hat{f}_{2}=\Gamma_{0} \hat{f}_{1}+\Gamma_{0}^{\prime} \hat{f}_{2}=0\right\} .
$$

For $\lambda \in \mathfrak{h}_{0}$ we have

$$
(\Theta-\widetilde{M}(\lambda))^{-1}=\left(\begin{array}{cc}
-(M(\lambda)+\tau(\lambda))^{-1} & (M(\lambda)+\tau(\lambda))^{-1} \tau(\lambda) \\
\tau(\lambda)(M(\lambda)+\tau(\lambda))^{-1} & M(\lambda)(M(\lambda)+\tau(\lambda))^{-1} \tau(\lambda)
\end{array}\right)
$$

and Theorem 2.2 implies

$$
(\widetilde{A}-\lambda)^{-1}=\left(\begin{array}{cc}
\left(A_{0}-\lambda\right)^{-1} & 0 \\
0 & \left(T_{1}-\lambda\right)^{-1}
\end{array}\right)+\widetilde{\gamma}(\lambda)(\Theta-\widetilde{M}(\lambda))^{-1} \widetilde{\gamma}(\bar{\lambda})^{+} .
$$

By our assumptions and the properties of $T_{0}$ the selfadjoint extension $A_{0} \times T_{0}$ of $A \times T$ in $\mathcal{H} \times \mathcal{K}$ is of type $\pi_{+}$over $\Omega^{\prime}$. Since the defect of $A_{0}$ and $T_{0}$ is one

$$
\left(A_{0} \times T_{0}-\lambda\right)^{-1}-(\widetilde{A}-\lambda)^{-1}, \quad \lambda \in \mathfrak{h}_{0},
$$

is a rank two operator. Making use of [2, Theorem 2.4] we conclude that $\widetilde{A}$ is of type $\pi_{+}$over $\Omega^{\prime}$.

Let us show that the compressed resolvent of $\widetilde{A}$ onto $\mathcal{H}$ is a solution of (4.3). By (4.6), (4.8) and (4.9) we obtain

$$
P_{\mathcal{H}}(\widetilde{A}-\lambda)^{-1} \mid \mathcal{H}=\left(A_{0}-\lambda\right)^{-1}-\gamma(\lambda)(M(\lambda)+\tau(\lambda))^{-1} \gamma(\bar{\lambda})^{+}
$$

for $\lambda \in \mathfrak{h}_{0}$. For a given $h \in \mathcal{H}$ we define

$$
f_{1}:=P_{\mathcal{H}}(\widetilde{A}-\lambda)^{-1}\{h, 0\} \quad \text { and } \quad f_{2}:=P_{\mathcal{K}}(\widetilde{A}-\lambda)^{-1}\{h, 0\} .
$$

Then

$$
\left(\begin{array}{c}
\left\{f_{1}, f_{2}\right\} \\
\left\{\lambda f_{1}+h, \lambda f_{2}\right\}
\end{array}\right) \in \widetilde{A}
$$

Since $\widetilde{A} \subset A^{+} \times T^{+}$we have $\hat{f}_{1}:=\left(\begin{array}{c}f_{1} \\ \lambda f_{1}+h\end{array}\right) \in A^{+}$and $\hat{f}_{2}:=\left(\begin{array}{c}f_{2} \\ \lambda f_{2}\end{array}\right) \in \hat{\mathcal{N}}_{\lambda, T^{+}},($see $(2.4))$. 
By (4.7), and since $\tau$ is the Weyl function of $\left\{\mathbb{C}, \Gamma_{0}^{\prime}, \Gamma_{1}^{\prime}\right\}$, we obtain

$$
\Gamma_{1} \hat{f}_{1}=\Gamma_{1}^{\prime} \hat{f}_{2}=\tau(\lambda) \Gamma_{0}^{\prime} \hat{f}_{2}=-\tau(\lambda) \Gamma_{0} \hat{f}_{1}
$$

for $\lambda \in \mathfrak{h}(\tau)$. We have shown that for $h \in \mathcal{H}$ and $\lambda \in \mathfrak{h}_{0}$ the vector $\hat{f}_{1}=\left(\begin{array}{c}f_{1} \\ \lambda f_{1}+h\end{array}\right) \in A^{+}$ is a solution of (4.3).

It remains to verify (4.5). Assume that the representation (3.2) is minimal (see (3.3)). Then, by (3.4),

$$
\operatorname{clsp}\left\{\gamma^{\prime}(\lambda) \mid \lambda \in \rho\left(T_{0}\right) \cap \Omega^{\prime}\right\}=\mathcal{K},
$$

and the set $\rho\left(T_{0}\right) \cap \Omega^{\prime}$ in (4.10) can be replaced by $\rho(\widetilde{A}) \cap \Omega^{\prime}$. From (4.6), (4.8) and (4.9) we obtain

$$
P_{\mathcal{K}}(\widetilde{A}-\lambda)^{-1}\{h, 0\}=\gamma^{\prime}(\lambda)(M(\lambda)+\tau(\lambda))^{-1} \gamma(\bar{\lambda})^{+} h
$$

for $h \in \mathcal{H}$ and $\lambda \in \mathfrak{h}_{0}$. If $h \notin \mathcal{N}_{\bar{\lambda}, A^{+}}^{[\perp]}$ we have $\gamma(\bar{\lambda})^{+} h \neq 0$. Making use of (4.10) we obtain

$$
\operatorname{clsp}\left\{P_{\mathcal{K}}(\widetilde{A}-\lambda)^{-1}\{h, 0\} \mid h \in \mathcal{H}, \lambda \in \rho(\widetilde{A}) \cap \Omega^{\prime}\right\}=\mathcal{K},
$$

and therefore (4.5) holds. Theorem 4.1 is proved.

Remark 4.3. Let $A \subset A_{0}$ and let $\left\{\mathbb{C}, \Gamma_{0}, \Gamma_{1}\right\}, \gamma$ and $M$ be as in the assumptions of Theorem 4.1. The case that $\tau \in N(\Omega)$ is a real constant is excluded in Theorem 4.1. In this case the boundary value problem (4.3) has the form

$$
f_{1}^{\prime}-\lambda f_{1}=h, \quad \tau \Gamma_{0} \hat{f}_{1}+\Gamma_{1} \hat{f}_{1}=0, \quad \hat{f}_{1}=\left(\begin{array}{c}
f_{1} \\
f_{1}^{\prime}
\end{array}\right) \in A^{+} .
$$

The relation $\widetilde{A}_{-\tau}=\operatorname{ker}\left(\tau \Gamma_{0}+\Gamma_{1}\right) \in \widetilde{\mathcal{C}}(\mathcal{H})$ (see $\left.(2.2),(2.3)\right)$ is a selfadjoint extension of $A$ in $\mathcal{H}$. By Theorem 2.2 we have

$$
\left(\widetilde{A}_{-\tau}-\lambda\right)^{-1}=\left(A_{0}-\lambda\right)^{-1}-\gamma(\lambda)(\tau+M(\lambda))^{-1} \gamma(\bar{\lambda})^{+}
$$

for $\lambda \in \mathfrak{h}(M) \cap \mathfrak{h}\left((\tau+M)^{-1}\right)$. Therefore, making use of the assumption that $A_{0}$ is of type $\pi_{+}$over $\Omega$ and [2, Theorem 2.4] we conclude that $\widetilde{A}_{-\tau}$ is also of type $\pi_{+}$over $\Omega$. Setting $f_{1}:=\left(\widetilde{A}_{-\tau}-\lambda\right)^{-1} h$ it follows that $\hat{f}_{1}:=\left(\begin{array}{c}f_{1} \\ \lambda f_{1}+h\end{array}\right) \in \widetilde{A}_{-\tau}$ is a solution of (4.11).

Remark 4.4. Let the assumptions be as in Theorem 4.1 and assume that $\widetilde{A}$ fulfils the minimality condition (4.5). Let $\widetilde{B}$ be a selfadjoint extension of $A$ in some Krein space $\mathcal{H} \times \widetilde{\mathcal{K}}$ which is of type $\pi_{+}$over $\Omega^{\prime}$ such that the compression of the resolvent of $\widetilde{B}$ onto $\mathcal{H}$ yields a solution of (4.3). Assume that $\widetilde{B}$ fulfils the minimality condition (4.5) with $\rho(\widetilde{A}) \cap \Omega^{\prime}$ replaced by $\rho(\widetilde{B}) \cap \Omega^{\prime}$. We denote the local spectral functions of $\widetilde{A}$ and $\widetilde{B}$ by $E_{\widetilde{A}}$ and $E_{\widetilde{B}}$, respectively (see (3.1)). Let $\Delta \subset \Omega^{\prime} \cap \overline{\mathbb{R}}$ be a closed connected set such that 
$E_{\widetilde{A}}(\Delta)$ is defined. Then also $E_{\widetilde{B}}(\Delta)$ is defined, the Pontryagin spaces $E_{\widetilde{A}}(\Delta)(\mathcal{H} \times \mathcal{K})$ and $E_{\widetilde{B}}(\Delta)(\mathcal{H} \times \widetilde{\mathcal{K}})$ have the same finite rank of negativity and the relations

$$
\widetilde{A}_{1}:=\widetilde{A} \cap\left(E_{\widetilde{A}}(\Delta)(\mathcal{H} \times \mathcal{K})\right)^{2} \quad \text { and } \quad \widetilde{B}_{1}:=\widetilde{B} \cap\left(E_{\widetilde{B}}(\Delta)(\mathcal{H} \times \widetilde{\mathcal{K}})\right)^{2}
$$

are unitarily equivalent (see [13]), that is, there exists an isometric isomorphism $V$ which maps $E_{\widetilde{A}}(\Delta)(\mathcal{H} \times \mathcal{K})$ onto $E_{\widetilde{B}}(\Delta)(\mathcal{H} \times \widetilde{\mathcal{K}})$ such that

$$
\left\{\left(\begin{array}{c}
V\{h, k\} \\
V\left\{h^{\prime}, k^{\prime}\right\}
\end{array}\right) \mid\left(\begin{array}{c}
\{h, k\} \\
\left\{h^{\prime}, k^{\prime}\right\}
\end{array}\right) \in \widetilde{A}_{1}\right\}=\widetilde{B}_{1} .
$$

\section{REFERENCES}

[1] T.Ya. Azizov, P. Jonas: On Locally Definitizable Matrix Functions, submitted.

[2] J. Behrndt, P. Jonas: On Compact Perturbations of Locally Definitizable Selfadjoint Relations in Krein Spaces, submitted.

[3] V.A. Derkach: On Weyl Function and Generalized Resolvents of a Hermitian Operator in a Krein Space, Integral Equations Operator Theory 23 (1995), 387-415.

[4] V.A. Derkach: On Generalized Resolvents of Hermitian Relations in Krein Spaces, J. Math Sciences 97 (1999), 4420-4460.

[5] V.A. Derkach, M.M. Malamud: Generalized Resolvents and the Boundary Value Problems for Hermitian Operators with Gaps, J. Funct. Anal. 95 (1991), 1-95.

[6] V.A. Derkach, M.M. Malamud: The Extension Theory of Hermitian Operators and the Moment Problem, J. Math. Sciences 73 (1995), 141-242.

[7] V.A. Derkach, S. Hassi, M.M. Malamud, H.S.V. de Snoo: Generalized Resolvents of Symmetric Operators and Admissibility, Methods of Functional Analysis and Topology 6 (2000), 24-53.

[8] A. Dijksma, H.S.V. de Snoo: Symmetric and Selfadjoint Relations in Krein Spaces I, Operator Theory: Advances and Applications 24 (1987), Birkhäuser Verlag Basel, 145-166.

[9] V.I. Gorbachuk, M.L. Gorbachuk: Boundary Value Problems for Operator Differential Equations, Kluwer Academic Publishers, Dordrecht (1991).

[10] P. Jonas: A Class of Operator-Valued Meromorphic Functions on the Unit Disc, Annal. Acad. Scie. Fenn. 17 (1992), 257-284.

[11] P. Jonas: Operator Representations of Definitizable Functions, Annal. Acad. Scie. Fenn. 25 (2000), 41-72.

[12] P. Jonas: On Locally Definite Operators in Krein Spaces, in: Spectral Theory and Its Applications, Theta, Bucharest (2003), 95-127.

[13] P. Jonas: On Operator Representations of Locally Definitizable Functions, submitted.

J. Behrndt and P. Jonas

Fachbereich Mathematik, MA 6 - 4

Technische Universität Berlin

Str. d. 17. Juni 136

10623 Berlin

Germany

E-mail: behrndt@math.tu-berlin.de jonas@math.tu-berlin.de 